\title{
Evaluación física y rehabilitación cardiovascular en niños con patología cardíaca
}

\author{
Emilio Covarrubias ${ }^{a}$, Cristián Clavería ${ }^{1}$.
}

\begin{abstract}
a Kinesiólogo Terapista Ventilatorio, Hospital Clínico Pontificia Universidad Católica de Chile.
1 Cardiólogo Pediatra, Facultad de Medicina, Departamento de Cardiología y Enfermedades Respiratorias, División de Pediatría. Pontificia Universidad Católica de Chile.
\end{abstract}

Declaración de conflictos de interés: Ninguno

Fuente de financiamiento: Ninguno

Resumen: Debido a los avances en las técnicas diagnósticas y resultados operatorios, los niños con cardiopatías congénitas (CC) tienen hoy mayor expectativa de vida. Nuestro hospital - un Centro de Referencia Nacional para la tratamiento de las CC en menores de 15 años -, ha reportado una disminución significativa de la mortalidad operatoria desde un $8,1 \%$ a un $4,7 \%$ entre 2000 y 2010 , pero ello no se ha acompañado de mejoría importante de la calidad de vida. La falta de programas de actividad física y par- ticipación social, puede ser un factor importante en la limitación de la calidad de vida. La evidencia existente describe que la medida referida produce mejorías en el consumo de oxígeno peak (VO2peak), tolerancia al esfuerzo físico y menores tasas de re hospitalización. En el presente artículo se revisan los métodos de evaluación de la condición física en la edad pediátrica, la evidencia actual de los programas de rehabilitación cardiovascular pediátrica y recomendaciones de actividad física en niños portadores de CC. 


\section{Physical evaluation and Cardiac rehabilitation in children with congenital heart disease}

Due to advances in diagnostic procedures and surgical results life expectancy of children with congenital heart disease (CHD) has increased significantly. In our hospital- a national reference center- operative mortality has decreased from $8.1 \%$ in 2000 to $4.7 \%$ in 2010. However, quality of life of these children has not improved accordingly. A significant factor which may account for this effect is the absence of physical activity and rehabilitation programs. Existing evidence points out to an increase in peak oxygen consumption, tolerance to physical exertion and a lower rate of rehospitalizations. Herein we review methods to evaluate physical condition in children, the current evidence supporting rehabilitation programs and the recommendations for physical activity in children with CHD. Keywords: congenital heart disease, rehabilitation, physical activity
Introducción: En la actualidad existe suficiente evidencia para establecer la relación entre actividad física y salud $^{1}$. La American Heart Association incluyó la inactividad física como un factor de riesgo de patología coronaria y enfermedades crónicas no transmisibles del adulto joven y de la tercera edad ${ }^{2}$. Debido a esto, el interés por la actividad física surge ya no como una necesidad personal, sino que como un objetivo de salud pública.

Estudios en población chilena describen altos índices de sedentarismo y obesidad ${ }^{3}$. El $88,6 \%$ de la población adulta es sedentaria (no realiza actividad física al menos 3 veces a la semana por $30 \mathrm{~min}$. $)^{3}$. En niños la obesidad va en alza alcanzando en menores de 6 años una prevalencia del 9,9\% y de sobrepeso del $22,4 \%{ }^{3}$. Estas condiciones determinan un aumento en aparición y desarrollo precoz de patologías cardiovasculares (arteriosclerosis, insuficiencia valvular, infarto agudo al miocardio, entre otras) $)^{3-4}$.
La prevalencia de niños portadores de cardiopatías congénitas (CC) varía entre 4 a 12 por 1000 recién nacidos vivos. Esta cifra se ha modificado, debido principalmente a un diagnóstico más certero y precoz ${ }^{5}$. El Hospital Clínico de la Pontificia Universidad Católica de Chile, que es uno de los centros de referencia nacional para la resolución quirúrgica de cardiopatías congénitas en menores de 15 años, describió una disminución significativa de su mortalidad operatoria de $8,1 \%$ a $4,7 \%$ en el período comprendido entre los años 2000 al $2010^{6}$. Hoy en día estos pacientes tienen una mayor expectativa de vida, debido al diagnóstico oportuno, avances en técnicas quirúrgicas, anestesia cardiovascular, soporte circulatorio y manejo pre y post operatorio en unidades de cuidados intensivos especializadas 6 .

Estos buenos resultados en la sobrevida de los pacientes no se acompañan siempre de una mejoría significativa en 
la calidad de vida y/o capacidad física ${ }^{7}$, lo que se atribuye a múltiples causas: cirugía paliativa, defectos anatómicos residuales, estadías hospitalarias prolongadas, tiempo de inactividad y desacondicionamiento físico y lo más frecuente, falta de programas de actividad física y participación social ${ }^{7-8-9}$. En resumen, estos pacientes son considerados por sus padres y el equipo de salud como "frágiles", restringiendo su participación en actividades físicas y sociales $^{7-8}$.

Se ha descrito que programas de rehabilitación cardiovascular en adultos con patología cardíaca aumentan su nivel de tolerancia al ejercicio, disminuyen índices de obesidad, mejoran los perfiles lipídicos, mejora el control glicémico y determinan una disminución en la mortalidad y morbilidad asociada ${ }^{9-10}$. Existen en la actualidad, pocos estudios en pacientes pediátricos portadores de $\mathrm{CC}$ que aborden el tema del incentivo de la actividad física y/o desarrollo de programas de rehabilitación cardiovascular ${ }^{10}$. Dupen et al, en una revisión sistemática de los efectos del entrenamiento físico en niños y adultos con patología cardíaca describió una mejoría en su tolerancia al ejercicio y consumo de oxígeno peak (VO2peak) sin registrar eventos adversos ${ }^{9}$. En Chile, el Ministerio de Salud desarrolló en el año 2005 y con posterior actualización el año 2010 la guía clínica Cardiopatías Congénitas Operables en menores de 15 años, la cual no hace referencia al desarrollo de programas de rehabilitación cardiovascular o estimulación de la actividad física. No se hace mención a la evaluación de la capacidad física o la presencia de factores de riesgo cardiovascular asociados y menos al desarrollo de programas de rehabilitación cardiovascular ${ }^{11}$, mientras que la población de niños con CC sigue en aumento.

\section{Evaluación de la condición física.}

La evaluación de la condición física del niño con patología cardíaca entrega información del estado del sistema cardiopulmonar bajo condiciones de exigencia y permite detectar la presencia de factores hemodinámicos que pudieran estar limitando su desempeño durante el ejercicio, como también identificar condiciones que impliquen riesgo de morbilidad asociada. Además, los cambios en peso - talla y de los sistemas musculo esquelético y cardiopulmonar en la edad pediátrica, generan un desafío mayor en la evaluación de este grupo de pacientes ${ }^{12-13}$.

Previo a la evaluación de la condición física, debe realizarse un análisis global del paciente que incluya: antecedentes clínicos, signos y síntomas según su patología de base, examen físico y evaluar si cumple con los criterios de inclusión para realizar test de esfuerzo (Tabla 1) ${ }^{14}$.
Tabla 1. Contraindicaciones absolutas y relativas para realizar el test de esfuerzo.

\section{ABSOLUTAS}

Proceso infeccioso agudo

Enfermedad cardíaca inflamatoria

Patología Respiratoria descompensada

Hipertensión arterial sistémica severa.

Daño ortopédico de algún grupo muscular.

Insuficiencia cardíaca congestiva

\section{RELATIVAS}

Obstrucción severa del flujo de salida del ventrículo izquierdo, de acuerdo a severidad.

Obstrucción severa del flujo de salida del ventrículo derecho, de acuerdo a severidad.

Insuficiencia cardíaca congestiva descompensada.

Enfermedad vascular pulmonar obstructiva.

Estenosis mitral severa.

Estenosis aórtica severa.

Enfermedad coronaria isquémica.

Miocardiopatía.

Síndromes de arritmia congénitos.

Arritmias ventriculares complejas, adquiridas.

\section{Tests e implementos para la evaluación de la condición física}

\section{Test de evaluación de la condición física. Test de marcha 6 minutos.}

Este test permite tener una visión real de la tolerancia al esfuerzo físico y la capacidad de realizar actividades cotidianas ${ }^{15}$. El paciente debe caminar la mayor distancia posible durante 6 minutos sobre una pista marcada de 30 metros. Durante este trayecto se monitoriza oximetría de pulso (SatO2), frecuencia cardíaca (FC), presión arterial (PA) y percepción del esfuerzo físico pre y post test. Los pacientes oxígeno dependientes deben usarlo durante el examen y es permitido detenerse para luego retomar el test. Esta prueba es fácil de realizar y económica. Sin embargo, la información que entrega no reemplaza al test de esfuerzo estándar ni permite hacer un diagnóstico de las causas que limitan el ejercicio. Lograr una mayor o menor distancia, también es dependiente de la motivación (operador y paciente), y de otros sistemas no relacionados con el sistema cardiovascular. A pesar de que se ha descrito mínimos efectos adversos, en pacientes sintomáticos este nivel de ejercicio con una limitada monitorización puede ser algo arriesgado $^{15}$. Se ha visto que la distancia alcanzada en 6 minutos, es un predictor de morbilidad y mortalidad en en- 
fermedades cardiopulmonares ${ }^{16}$.

Para obtener una correcta interpretación de los resultados, se deben considerar factores como: edad, peso, talla, género y patología subyacente ${ }^{14}$. Algunos valores promedios obtenidos de investigaciones, muestran distancias alcanzadas para niños sanos entre 7 a 16 años de 642 metros en mujeres y 680 metros en hombres ${ }^{16}$.

\section{Test de la lanzadera}

Es una prueba de carga incremental, el paciente debe desplazarse caminando entre 2 conos separados por 10 metros a un ritmo que aumenta gradualmente, de acuerdo a una grabación preestablecida, hasta completar los 12 niveles que incluye el test. Los parámetros que se evalúan son la FC, SatO2 y percepción de esfuerzo previo al inicio, entre cada nivel finalizado y al término de la prueba. El test se debe detener por 3 razones: el paciente refiere que no puede mantener el ritmo, no cumple con la distancia entre los conos por cada vuelta y si se alcanza el $85 \%$ de la frecuencia cardíaca máxima teórica (FCMT) ${ }^{17}$.

Este test se ha utilizado en estudios de evaluación de la capacidad cardio-respiratoria en niños obesos, con patología respiratoria crónica como displasia broncopulmonar y fibrosis quística, y niños y adultos en tratamiento contra el cáncer ${ }^{17}$.

\section{Test de Naveta}

Evalúa la máxima capacidad aeróbica del sujeto basada en un protocolo incremental e indirecto. Puede estimar el consumo de oxígeno máximo (VO2max) usando ecuaciones de regresión propuestas por Léger, en base a la velocidad alcanzada entre cada etapa y la edad del sujeto ${ }^{18}$. Consiste en que el paciente se mueve en función de una señal sonora entre 2 conos separados por 20 metros y el ritmo de carrera va aumentando progresivamente según dicha señal. Los parámetros que se controlan son: FC inicial y final, SatO2 y número de etapas alcanzadas. La detención del test ocurre cuando el sujeto no pueda mantener el ritmo entre cada etapa ${ }^{18}$. La confiabilidad y validez de este test para su uso en niños y adolescentes ha sido ampliamente demostrada $^{19}$. Su mayor utilidad se describe como parte de la evaluación de la condición física en niños y adolescentes sedentarios, obesos y adultos con cáncer ${ }^{20-21-22}$. No existen reportes de su uso en niños con cardiopatías congénitas.

\section{Test de esfuerzo}

Es una prueba que evalúa la tolerancia al ejercicio y su respuesta cardiovascular llegando a un nivel de alta inten- sidad (pudiendo alcanzar 70\% - 90\% de la FCMT) ${ }^{23}$. En la población pediátrica con patología cardíaca las indicaciones para el test son: evaluar la tolerancia al esfuerzo físico, obtener información de la respuesta cronótropa, presora, presencia de arritmias o signos de isquemia, para complementar información clínica y de imágenes, para ayudar a decidir el tipo de terapia de cada paciente (cirugía, medicamentos y/o rehabilitación). Permite, además, evaluar la respuesta farmacológica (b-bloqueantes, inhibidores de la enzima convertidora de angiotensina (IECA), digoxina, entre otros y evalúa potenciales condiciones de riesgo cardiovascular ${ }^{24-25}$.

Durante el test se monitoriza el trazado electrocardiográfico basal y su respuesta en ejercicio y etapa de recuperación, para detectar alteraciones eléctricas (cambios del segmento ST, onda T e intervalo QT) y evidenciar la presencia de arritmias. En forma paralela se debe evaluar la SatO2, PA y percepción subjetiva del esfuerzo. Toda esta monitorización debe realizarse previo, durante el test y en el período de recuperación ${ }^{25}$.

A la prueba de esfuerzo se puede complementar el aporte de oxígeno a través de una mascarilla con sensor de flujo proximal, lo que permite analizar gases inspirados y espirados durante el test. Esta técnica se conoce como ergoespirometría, que consiste en el análisis del oxígeno consumido y dióxido de carbono espirado evaluando la capacidad del sistema cardiopulmonar para mantener un adecuado aporte de oxígeno hacia los tejidos ${ }^{23}$. Por medio de esta técnica, se logra medir el consumo de oxígeno máximo y peak (VO2max y VO2peak) y consumo de dióxido de carbono (VCO2), permitiendo calcular el valor de la tasa de intercambio respiratorio (RER), usado para evaluar la cantidad de sustrato energético que utiliza el paciente al realizar ejercicio ${ }^{23}$.

El VO2 es influenciado por la edad, género, factores genéticos, hábitos de ejercicio, y estado funcional del sistema cardiovascular. Se ha descrito en estudios poblacionales que a menor VO2, menor respuesta en FC (cronotropismo negativo), así también cuando mayor es la relación volumen exhalado/consumo de dióxido de carbono (VE/ VCO2), mayor es la mortalidad ${ }^{23}$.

Para la prueba de esfuerzo, existen distintos protocolos para evaluar el sistema cardiopulmonar. En general se dividen en 3 grandes grupos: (a) carga incremental tipo escalera; aumenta pendiente y velocidad o resistencia con una fase de meseta entre cada incremento, son empleados para evaluar el VO2máx o VO2peak y se puede emplear este esquema en treadmill, con los protocolos de Bruce, Bruce modificado y Balke, o cicloergómetro, con los protocolos 
de James y McMaster, (b) trabajo en rampa; se aumenta la intensidad y resistencia simultáneamente de forma progresiva y (c) trabajo constante; se emplea una carga constante, en un tiempo variable 23-24. $^{2}$.

Se han establecido criterios de detención del test, que se basan en criterios; clínicos, alteraciones del ECG y PA (Tabla 2) ${ }^{24}$.

Tabla 2. Criterios de detención en un test cardiopulmonar para niños.

\begin{tabular}{|c|c|}
\hline Criterios & Signos \\
\hline \multirow[t]{2}{*}{ - Clínicos } & $\begin{array}{l}\text { *Síntomas de dolor torácico, dolor de cabeza intenso, } \\
\text { disnea, nausea, somnolencia, palidez, desorientación. }\end{array}$ \\
\hline & *Paciente solicita terminar el estudio. \\
\hline \multirow[t]{6}{*}{ - ECG } & $\begin{array}{l}\text { *Corazón no aumenta la FC durante el ejercicio, } \\
\text { extrema fatiga y disnea. }\end{array}$ \\
\hline & $\begin{array}{l}{ }^{*} \text { Contracción prematura ventricular, con aumento } \\
\text { de la FC. }\end{array}$ \\
\hline & *Alteraciones del ritmo. \\
\hline & *Supradesnivel del ST > 3mm \\
\hline & ${ }^{*}$ Bloqueo aurículoventricular, $2^{\circ}$ grado. \\
\hline & *Segmento QT > 500ms. \\
\hline \multirow[t]{3}{*}{ - Presión arterial } & ${ }^{*} \mathrm{PA}>250 / 125 \mathrm{mmHg}$. \\
\hline & $\begin{array}{l}\text { *Progresiva disminución de la presión arterial } \\
\text { sistólica (PAS), con mayor carga de trabajo. }\end{array}$ \\
\hline & *Brusco descenso en la SatO2. \\
\hline
\end{tabular}

\section{Implementos.}

Para la evaluación cardiopulmonar se debe tener: un espacio con adecuadas condiciones de temperatura y humedad, un equipo de profesionales con experiencia en la evaluación de esta población, y estar implementado con un sistema de respuesta rápida frente a situaciones de emergencia que incluya: carro de paro con desfibrilador, insumos y fármacos para reanimación cardiopulmonar avanzada, redes de gases clínicos (oxígeno y aire) y contar siempre con la presencia de un cardiólogo con experiencia en reanimación cardiopulmonar básica durante el desarrollo del test de esfuerzo ${ }^{26}$.

Para realizar el ejercicio se debe contar con ergómetros, cicloergómetro o treadmill. Ambos han sido usados en niños, sin embargo, se describe que las mediciones de VO2peak con treadmill arrojan valores mayores en un 5\% a $10 \%$ que los obtenidos con el cicloergómetro, debidos al uso de mayor cantidad de grupos musculares ${ }^{26}$. El treadmill es más apropiado para niños, ya que solo involucra mantener un ritmo sobre la cinta siendo más fácil su adaptación. La cantidad de trabajo depende del peso corporal, velocidad de la cinta y pendiente programada, siendo la fatiga de extremidades inferiores la principal causa de tér- mino del test ${ }^{26}$.

El cicloergómetro se recomienda para uso en niños mayores a $125 \mathrm{~cm}$, requiere una práctica y aprendizaje de coordinación con los pedales, el peso del paciente no es una variable que afecte la resistencia en gran medida durante el pedaleo y el tronco superior está más estable que en el treadmill, lo que permite realizar mejores registros del electrocardiograma, FC y PA durante el test ${ }^{26}$.

Para el análisis de los gases se debe contar con un sistema de evaluación de consumo de oxígeno (Figura 1), que incluya un software computacional de análisis de gases, sensor de flujo proximal al paciente y gases de calibración ${ }^{23}$.

\section{Programa de rehabilitación cardiovascular.}

El programa de rehabilitación cardiovascular viene a revertir, en lo posible, los efectos de la inactividad y el desacondicionamiento físico. Estudios realizados en adultos con patología cardíaca han mostrado resultados beneficiosos en términos de: perfil lipídico, disminución de la obesidad y mejoría en la capacidad física ${ }^{1}$.

Ubeda A. et al, realizó una revisión sistemática acerca de los Programas de Rehabilitación Cardíaca en niños y observó consenso en la duración de los programas, los cuales deben ser de al menos de 12 semanas, con una frecuencia de 2 a 3 veces por semana, con sesiones de $40 \mathrm{~min}$. Sin embargo, no hay acuerdo sobre los esquemas de rehabilitación y los test de evaluación a utilizar. Los mejores resultados se han observado al combinar ejercicios aeróbicos, de resistencia y flexibilidad, sumado a una intervención sicológica y exigiendo una intensidad del ejercicio de 70 $80 \%$ de la $\mathrm{FCMT}^{7}$.

Rhodes J. et al. desarrollaron un programa de entrenamiento cardiovascular de 1 hora de duración, 2 veces a la semana, durante un período de 12 semanas a 16 pacientes post cirugía cardíaca. Observaron un aumento del VO2 peak y de la carga de trabajo tolerada. La evaluación posterior al mismo grupo de pacientes demostró que los efectos beneficiosos se mantenían luego de 6 meses post rehabilitación, en términos de VO2 peak y tolerancia a la carga de trabajo. Estos resultados se correlacionan con la mejoría en autoestima, comportamiento y estado emocional observados ${ }^{10-27}$.

Duppen y et al., realizaron una revisión sistemática de los programas de entrenamiento físico para niños y adultos con cardiopatías congénitas, que incluyó 31 artículos, en 621 pacientes (edades entre 4 años y 45 años), Los programas de entrenamiento tuvieron una duración de 12 semanas con frecuencia de ejercicio 3 veces por semana y los diagnósticos asociados más frecuentes fueron estado 


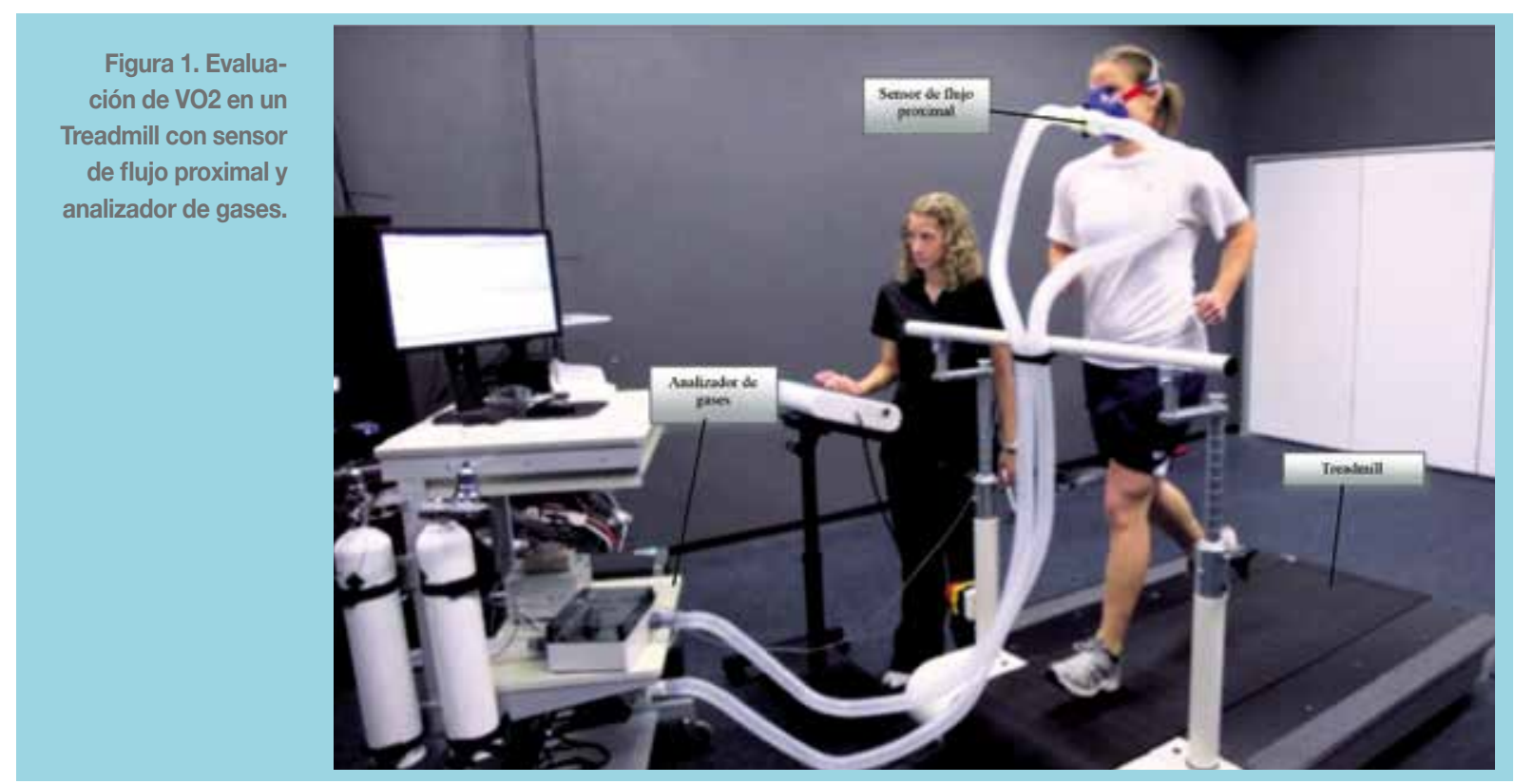

postoperatorio de cirugía correctora de tetralogía de Fallot y Fontan. Ellos encontraron que el VO2 máx., nivel de actividad física, tolerancia al esfuerzo físico y fuerza muscular mejoraron significativamente y no se reportaron efectos adversos relacionados con el ejercicio. No se pudo establecer con claridad las diferencias entre el potencial de entrenamiento de distintas cardiopatías, porque en cada estudio analizado se agrupaban diversas patologías y la muestra por cada una de ellas era reducida. La severidad de las secuelas posterior a la cirugía y su consecuente repercusión hemodinámica podrían tener un fuerte impacto en el desempeño del ejercicio y los resultados en el programa de entrenamiento 9 .

La revisión de la literatura internacional muestra que los programas de rehabilitación cardíaca pediátrica son escasos pero, las diferentes publicaciones coinciden que la rehabilitación cardiovascular tiene un gran potencial en el beneficio y en el manejo integral de los pacientes con patología cardíaca congénita. La escasa experiencia internacional y nula disponibilidad de estos programas a nivel nacional ha hecho que los beneficios de estos programas no estén disponibles para la mayoría de los niños con patología cardíaca en nuestro país. Como se sugiere en reportes de consenso, el entrenamiento basado en el ejercicio físico debe ser parte del esquema de tratamiento de los niños con cardiopatías congénitas ${ }^{28,29}$.

\section{Ejercicio en pacientes con cardiopatías congénitas.}

Todos los niños tienen la necesidad de moverse, jugar y desarrollar distintos tipos de actividades; sin embargo, en el grupo de niños con patología cardíaca aún existe mucha incertidumbre sobre la mejor recomendación del tipo de ejercicio a realizar ${ }^{30}$.

En general, todas las sesiones de ejercicio y entrenamiento deben comenzar con 10 - 15 minutos de calentamiento, seguido de 20 - 60 minutos del ejercicio principal y finalmente 10 minutos de vuelta a la calma. En los niños el desafío principal es mantener la motivación por el ejercicio, que va cambiando según la edad. En edad prepuberal el objetivo del ejercicio se basa en mejorar la eficiencia del control neuromotor de la musculatura y no en mejorar la masa muscular; por lo cual, se debe dar énfasis a las actividades relacionadas con la coordinación, teniendo precaución en el entrenamiento de la fuerza y flexibilidad. En niños púberes el componente social juega un rol importante; ser parte de un equipo y cumplir con normas son aspectos relevantes a considerar. Después de la pubertad el entrenamiento puede ser "similar" al de los adultos, enfocándose en la fuerza (masa muscular) y resistencia (capacidad aeróbica), incluyendo siempre los aspectos ya mencionados (coordinación, flexibilidad y habilidades sociales). En todos los grupos mencionados una de las claves en el ejercicio es que sea entretenido ${ }^{30-31}$.

El ejercicio, por sí sólo y en especial para niños con patología cardíaca, busca generar un estilo de vida saludable y en el largo plazo prevenir la aparición de enfermedades crónicas no transmisibles, mejorando la sobrevida de estos pacientes ${ }^{29-30}$. 


\section{Conclusiones:}

En Chile se ha demostrado una disminución significativa de la mortalidad operatoria en la resolución quirúrgica de las CC en menores de 15 años, lo que ha mejorado las expectativas de vida. Sin embargo, no existen estudios nacionales que evalúen la relación existente entre estos buenos resultados quirúrgicos, calidad de vida y condición física. A nivel internacional se destaca la utilidad de la práctica deportiva y desarrollo de programas de rehabilitación cardiovascular en pacientes pediátricos portadores de CC con resultados alentadores ${ }^{30}$. La Sociedad de Cardiología Europea publicó el año 2013, recomendaciones muy prác- ticas en relación al desarrollo de programas de actividad física para adolescente y adultos con cardiopatía congénita, basado principalmente en variables hemodinámicas y electrofisiológicas más que en el tipo de defecto cardíaco, permitiendo así prescribir la actividad física en forma individual $^{29}$.

El desafío actual es investigar cómo la fisiología del ejercicio en estos sujetos se adapta a la actividad física y así desarrollar, implementar y evaluar los resultados de un Programa de Rehabilitación Cardiovascular en el grupo de pacientes portadores de CC , como también su impacto en su calidad de vida, en nuestro país.

\section{Referencias:}

1. THOMSON P., BUCHNER D., PIÑA I., Exercise and physical activity in the prevention and treatment of atherosclerotic cardiovascular disease. Circulation, 2003; 107: 3109 - 3116.

2. STRATH S., KAMINSKY L., AINSWORTH B., Guide to assessment of physical activity: clinical and research applications. Circulation. 2013; 128: 2259 - 2279.

3. ENCUESTA NACIONAL DE SALUD. 2010, MINSAL. http://web.minsal.cl/portal/url/item/bcb03d7bc28b64dfe040010165012d23.pdf (accedido 10 agosto de 2014).

4. INDICADORES DE OBESIDAD EN POBLACIÓN CHILENA, 2010, MINSAL. http://web.minsal.cl/portal/url/item/9ad9cbfb71ca4705e04001011e010283.pdf (accedido 2 de noviembre de 2014).

5. VAN DER LINDE D., KONINGS E., SLAGER M., BIRTH prevalence of congenital heart disease worldwide, a Systematic Review and Meta-Analysis. J Am CollCardiol. 2011; 58: 2241 $-2247$.

6. CLAVERIA C., CERDA J., BECKER P., Mortalidad operatoria y estratificación de riesgo en pacientes pediátricos operados de cardiopatía congénita: experiencia de 10 años. Rev Chil Cardiol. 2014; 33: 11 - 19.

7. UBEDA A., RODRIGUEZ A., ARROYO O., Pediatrics cardiac rehabilitation in congenital heart disease. A systematic review. Cardiology in the young. 2012; 22: $241-250$.

8. BUDTS W., BORJESSON M., CHES. Invidualizaed exercises
M., Physical activity in adolescents and adults with congenital $\mathrm{H}$ heart disease: indivualized exercise prescription. European Heart journal. 2013, 34: 3669 - 3674.

9. DUPPEN N., TAKKEN T., HOPMAN M.T.E., Systematic review of the effects of physical exercise training programmes in children and young adults with congenital heart dise ase . International Journal of Cardiology. 2013; 168: 1779 - 1787.

10. RHODES J., CURRAN T., CAMIL L., Impact of Cardiac Rehabilitation on the Exercise Function of Children with serious Congenital Heart disease. Pediatrics. 2005; 116: 1339 - 1345.

11. GUÍA CLÍNICA. Cardiopatías Congénitas operables en menores de 15 años. 2010,MINSAL.http://web.minsal.cl/portal/url/ item/720bfefe91e0d2ede04001011f010ff2.pdf (accedido el 4 de agosto del 2014).

12. RHODES J., TIKKANEN A., JENKINS J., Exercise testing and training in children with congenital heart disease. Circulation. 2010; 122: 1957 - 1967.

13. TAKKEN T., BLANK A.C., HULZEBOS E.H., Cardiopulmonary Exercise testing in congenital Heart disease: (contra) indications and interpretation. Neth Heart J. 2009; 17: 385 - 392.

14. PARIDON S., ALPERT B., BOAS S., Clinical Stress Testing in the Pediatric Age Group, a statement from the American Heart Association Council on Cardiovascular Disease in the Young, Committee on Atherosclerosis, Hypertension, and Obesity in the Youth. Circulation. 2006; 113: 1905-1920. 
15. ATS STATEMENT: Guidelines for the Six-Minute walk test. American Journal of Respiratory and Critical Care of Medicine. 2002 ; 166: $111-117$.

16. ALBERT M., YIN J., AU J., Standard reference for the six-minute-walk test in healthy children aged 7 to 16 years. American Journal of Respiratory and Critical Care of Medicine. 2007; 176: $174-180$

17. SINGH S., MORGAN M., SCOTT S., Development of a shuttle walking test of a disability in patients with chronic airways obstruction. Thorax. 1992; 47:1019 - 1024.

18. MELO X., SANTA-CLARA H., ALMEIDA J., Comparing several equations that predict peak VO2 using the 20-m multistage- shuttle run-test in 8-10-year-old children. Eur J Appl Physiol. 2011. 111: 839 - 849.

19. LÉGER L., LAMBERT J., A maximal multistage 20-m shuttle runt test to predict VO2max. Eur J Appl Physiol. 1982; 49: 1 - 12.

20. ORTEGA F., RUIZ J., CASTILlO M., Physical fitness in childhood and adolescence: a powerful marker of health. International Journal of Obesity. 2008. 32; 1- 1.

21. ORTEGA F., RUIZ J., CASTILLO M., Low level of physical fitness in Spanish adolescents. Relevance for future cardiovascular health. Rev Esp Cardiol. 2005; 58: 898 - 909.

22. QUINART S., MOUGIN F., SIMON-RIGAUD M., Evaluation of cardiorespiratory fitness using three field tests in obese adolescents: Validity, sensitivity and prediction of peak VO2.J SciMed Sport. 2014 Sep; 17: 521 - 525.

23. FORMAN D., MYERS J., LAVIE C., Cardiopulmonary exercise testing: relevant but underused. Postgraduate Medicine. 2010; 112: 68 - 86.Takken T., Blank A.C., Hulzebos E.H, Cardiopulmonary exercise testing in congenital heart disease: equipment and test protocols. Netherlands Heart Journal. 2009; 17: $339-344$.
24. TAKKEN T., BLANK A., HULZEBOS E., Cardiopulmonary testing in congenital heart disease: (contra) indications and interpretation. Netherland heart journal. 2009; 17: 385 - 392.

25. PARIDON S., ALPERT B., BOAS S., Clinical stress test in the pediatric age group. Circulation. 2006; 113: 1905 - 1920.

26. TAKKEN T., BLANK A., HULZEBOS E., Cardiopulmonary exercise testing in congenital heart disease: equipment and test protocols. Netherland heart journal. 2009; 17: 339 - 344 .

27. RHODES J., CURRAN T., CAMIL L., Sustained effects of cardiac rehabilitation in children with serious congenital heart disease. Pediatrics. 2006; 118: 586 - 593.

28. GOMES-NETO M, SAQUETTO MB, DA SILVA E SILVA CM, CONCEIÇÃO CS, CARVALHO VO. Impact of exercise training in aerobic capacity and pulmonary function in children and adolescents after congenital heart disease surgery: a systematic review with meta-analysis. Pediatr Cardiol. 2015.

29. BUDTS W., BORJESSON M., CHES. Invidualizaed exercises M., Physical activity in adolescents and adults with congenital $\mathrm{H}$ heart disease: indivualized exercise prescription. European Heart journal. 2013, 34: 3669 - 3674 .

30. TAKKEN T.,GIARDINI A., REYBROUCK T., Recommendations for physical activity, recreation sport, and exercise training in paediatric patients with congenital heart disease: a report from the Exercise , Basic \& Trasnslational Research Section of the European Congenital Heart and Lung Exercise Group, and the Association for European Pediatrics Cardiology. European Journal of Preventive Cardiology. 2011; 19: 1034 -1065 .

31. MARON B., ZIPES D., ACKERMAN M., 36th Bethesda Conference: Elegibility recommendations for competitive athletes with cardiovascular abnormalities. JACC. 2005; 45: 1313 1375. 